\title{
Improving and standardizing capture of pediatric cardiac surgical complications
}

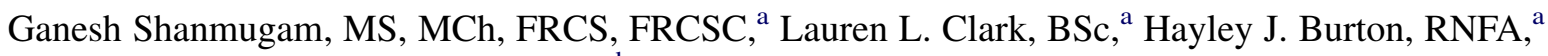

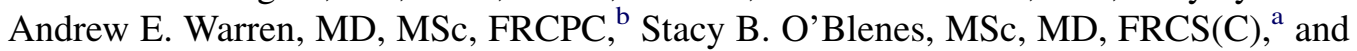 \\ Camille L. Hancock Friesen, MSc, MD, FRCS(C), FACS ${ }^{\mathrm{a}}$
}

\begin{abstract}
Objective: Our objective was to establish baseline data and develop a tool to allow for systematic evaluation of pediatric cardiac surgical complications. As a first step, we examined the incidence and distribution of complications, risk stratified by case complexity in a single institution. With improving mortality rates for congenital heart surgery, the next frontier for improving patient outcomes is characterizing and reducing complications. Currently, no standardized approach is available to monitor the incidence and severity of all complications associated with a congenital cardiac surgery program.
\end{abstract}

\begin{abstract}
Methods: Complications occurring in pediatric cardiac surgical patients (January 2006 to March 2009) were collected by database review applying standardized definitions. The surgical procedures were stratified by complexity to analyze the distribution of complications over the risk spectrum. Each complication was assigned a severity coefficient (1-3) used to calculate the combined effect of frequency and severity. The cumulative sum method was used to determined the trend of the adverse outcomes.
\end{abstract}

Results: Of 292 procedures, $84(28.8 \%)$ were associated with a total of 150 complications. Of the 150 complications, 37 occurred in patients who died. The most common complications were arrhythmias $(14.5 \%)$, cardiac $(12.6 \%)$, and operative $(12.6 \%)$. There was a linear relationship between the frequency and severity of complications and surgical complexity, as stratified using the Risk Adjustment for Congenital Heart Surgery category or Aristotle basic complexity levels (Spearman's coefficient $=1$ ).

Conclusions: When examined in a systematic fashion, the risk of complications in pediatric cardiac surgical patients is considerable. Our data illustrate that it is possible to track complications over time in a consistent manner. The effect of complication monitoring on patient outcomes remains to be proved. (J Thorac Cardiovasc Surg 2012;144:570-6)

Pediatric cardiac surgery is a discipline in evolution, with progressively improving mortality rates. As public demand for institutional reporting of outcomes has grown, the profession has searched for accurate and objective measures of program performance. Mortality is a well-defined, universally captured, and readily measured outcome. The reporting and comparison of institutional outcomes, to date, has focused on overall operative mortality, currently $4 \%$ in large multi-institutional data sets. ${ }^{1}$ However, a large proportion of adverse outcomes are attributable to nonfatal complications that are not captured in mortality reports. Morbidity is defined as "a state of illness or lack of health that includes physical, mental or emotional disability" by the Multi-Societal Committee for Pediatric and Congenital

From the IWK Division of Cardiac Surgery ${ }^{\mathrm{a}}$ and IWK Division of Cardiology, ${ }^{\mathrm{b}}$ Dalhousie University, Halifax, Nova Scotia, Canada.

Disclosures: Authors have nothing to disclose with regard to commercial support. Received for publication June 17, 2011; revisions received Jan 13, 2012; accepted for publication Jan 25, 2012; available ahead of print Feb 27, 2012.

Address for reprints: Camille L. Hancock Friesen, MSc, MD, FRCS(C), FACS, IWK Health Centre, 5850/5980 University Avenue, Halifax, NS B3K 6R8 Canada (E-mail: camillehf@hotmail.com).

0022-5223/\$36.00

Copyright (c) 2012 by The American Association for Thoracic Surgery doi:10.1016/j.jtcvs.2012.01.070
Heart Disease. ${ }^{2}$ Hence all operations are accompanied by some degree of morbidity. A complication, however, is defined as "an event or occurrence that is associated with disease or a healthcare intervention that is (a) a departure from the desired course of events and (b) may cause or be associated with suboptimal outcome."2 Complications can negatively affect patient outcomes, including length of stay in the intensive care and hospital and long-term quality of life. ${ }^{3}$ Complications can also be associated with an increased cost to the healthcare system. Although in some reports of adult cardiac surgery, complications are associated with mortality, ${ }^{4}$ there is evidence to suggest that complications might reflect something different to mortality and could be worth examining as a separate indicator of program performance. For example, Silber and colleagues ${ }^{5}$ reported that the hospital characteristics associated with mortality were not associated with complications. Shroyer and associates $^{6}$ also reported no significant correlation between riskadjusted mortality and risk-adjusted complications.

Although many centers collect and report series of pathology-specific or case-specific complication data, no systematic method of reporting the incidence or distribution of complications for an entire surgical program is available. Specifically, no benchmarks are in use for intra- or 


\section{Abbreviations and Acronyms \\ $\mathrm{ABC}=$ Aristotle basic complexity \\ CUSUM $=$ cumulative sum \\ ECMO = extracorporeal membrane oxygenation \\ RACHS $=$ Risk Adjustment for Congenital Heart Surgery}

interinstitutional comparison of risk-adjusted complications, even though up to $30 \%$ of congenital heart surgery admissions can be associated with complications. ${ }^{7}$ An important step toward the meaningful collection of complication data was the Multi-Societal Committee for Pediatric and Congenital Heart Disease definition list of surgical complications. $^{2}$

The objective of the present study was to report the frequency of complications in a retrospective singleinstitution pediatric cardiac surgical cohort using standardized definitions. Our secondary objective was to develop a complication reporting system to accurately capture pediatric cardiac surgery complications and to examine the incidence and distribution of complications, stratified by surgical case complexity. Rather than trying to provide specific complication data for a given operation or diagnosis, we have attempted to develop a tool that will allow programs to monitor their own outcomes in real time, to be able to respond quickly to any changes in the incidence of complications, and to thus enhance patient outcomes.

\section{METHODS}

The research ethics board of the IWK Health Centre approved the retrospective cohort analysis of complications occurring in congenital cardiac surgical procedures from January 1, 2006, to March 31, 2009. The IWK Health Centre is the tertiary referral center for Atlantic Canada and is a full-service cardiac surgical center, with the exception of cardiac transplantation and long-term ventricular assist device implantation. All cardiac surgical procedures performed during the study period were included in the analysis, with the exception of surgical closure of patent ductus arteriosus in premature neonates, primary extracorporeal membrane oxygenation (ECMO) and cardiac pacemaker and defibrillator implantations. Complications were collected by database review using the short list of 52 complications as defined by the Multi-Societal Committee for Pediatric and Congenital Heart Disease. ${ }^{2}$ Complications were identified by 1 clinic nurse on a quarterly basis by scanning each patient chart and identifying the complications recorded in the progress notes and discharge summaries (completed contemporaneously with patient discharge by the attending pediatric cardiologist and dictated by the attending pediatric cardiac surgeon). On a quarterly basis, the pediatric cardiologists and cardiac surgeons met and reviewed each of the patients and all the complications. Any discussion regarding complication identification or reclassification led to additional chart review and a final record of the complications was made. On an annual basis, the entire cohort and all the complications were reviewed numerically to inform the group of the overall program performance. Complications that did not fit into the standardized definition list were included under the heading "other complications."

The surgical procedures were risk stratified according to surgical complexity using either the Risk Adjustment for Congenital Heart Surgery $(\mathrm{RACHS})^{8}$ or Aristotle basic complexity (ABC) scores. ${ }^{9}$ The RACHS category is an expert consensus ranking of 79 surgical procedures that was developed as a risk-adjustment tool according to the differences in surgical complexity and validated for comparing mortality outcomes. The $\mathrm{ABC}$ score is determined by expert consensus of surgical complexity and the likelihood of complications. The surgical complexity score was assigned according to the highest risk procedure in the event that an operation involved 2 or more procedures performed concurrently.

Mortality was classified as intraoperative or postoperative, including all deaths occurring within 30 days postoperatively or during the index hospitalization. To factor in the severity of complications to our model, each complication was weighted by assigning a severity coefficient (range, $1-3$ ), according to expert consensus, ranging from the lowest severity (severity coefficient 1 , defined as mild or temporary deficit, predicted to minimally affect the patient's course and outcome) to the greatest severity (severity coefficient 3 , defined as a severe and/or permanent complication predicted to significantly impair the patient's outcome and possibly precede death). For calculations in which patients experiencing mortality were included in the analysis, death was assigned a severity coefficient of 5. Because of the debate surrounding the inclusion of "mortality" as a complication, we included death as a category of complication for the first part of the analysis and excluded cases with mortality for the second part of the analysis. Complications categorized as "other" were not assigned a severity coefficient.

To determine the total magnitude of complications (a variable we have termed "morbidity burden") in a single surgical complexity stratum, the weighted complications were stratified by RACHS category or ABC level and then summed (morbidity burden $=\Sigma$ [frequency $\times$ severity]) for each procedure in a particular category. RACHS categories 5 and 6 were collapsed into a single category owing to the small number of procedures in each category. Because of the unequal distribution of procedures across the RACHS categories (Table 1), the morbidity burden was divided by the number of procedures in the corresponding surgical stratum to generate a variable we termed "indexed morbidity," which is a reflection of the average morbidity burden per procedure in each risk category (indexed morbidity $=$ morbidity burden/number of procedures in category).

The cumulative sum (CUSUM) method was applied to track adverse outcomes over time. To construct a CUSUM plot, consecutive cases were plotted as a single unit on the $\mathrm{x}$ axis and the occurrence of any adverse event was plotted by the movement of 1 unit on the $y$ axis. The construction of alarm limits on the CUSUM curves is possible using the technique described by Rogers and colleagues. ${ }^{10}$ The following values were used to construct the alarm limits. First, using our retrospective data, the "acceptable" morbidity rate was set at $28.8 \%$. Second, the unacceptable morbidity rate was set at 1.5 times the acceptable mortality rate $(43.2 \%)$. Third, the type $1(\alpha)$ error rate was set at $5 \%$. Finally, the type $2(\beta)$ error rate was set at $5 \%$.

\section{Statistical Analysis}

The raw frequency data were obtained for each complication. We compared the incidence and severity of the complications separately in the survivors and deceased patients. A CUSUM plot was derived for the complications of the entire cohort.

\section{RESULTS \\ Demographics}

From January 1, 2006 to March 31, 2009, 268 patients (median age, 178 days; range, 1 day to 18 years) underwent 292 procedures (Table 1). Of the 268 patients, 50 were neonates $(17.1 \%)$ and 132 were infants $(45.9 \%)$. Most (205/ 292) procedures were RACHS category 2 or 3 . Procedures performed 5 or more times accounted for $208(71 \%)$ of the 292 procedures. 
TABLE 1. Demographics

\begin{tabular}{|c|c|c|c|c|c|c|}
\hline $\begin{array}{c}\text { RACHS } \\
\text { level }\end{array}$ & $\begin{array}{c}\text { Procedures } \\
\text { (n) }\end{array}$ & $\begin{array}{c}\text { Complications } \\
\text { (n) }\end{array}$ & $\begin{array}{l}\text { Procedures with } \\
\text { complications (n) }\end{array}$ & $\begin{array}{c}\text { Procedures with } 1 \\
\text { morbidity (n) }\end{array}$ & $\begin{array}{c}\text { Procedures with multiple } \\
\text { morbidities (n) }\end{array}$ & Mortality (n) \\
\hline 1 & 57 & 6 & 6 & 6 & 0 & 0 \\
\hline 2 & 115 & 36 & 23 & 16 & 7 & 1 \\
\hline 3 & 90 & 72 & 39 & 23 & 16 & 5 \\
\hline 4 & 12 & 13 & 8 & 5 & 3 & 0 \\
\hline 5 and 6 & 18 & 23 & 8 & 3 & 5 & 3 \\
\hline Total & 292 & 150 & 84 & 53 & 31 & $9(3.1)$ \\
\hline
\end{tabular}

Data in parentheses are percentages. RACHS, Risk Adjustment for Congenital Heart Surgery.

\section{Mortality}

The overall procedural mortality was $3.1 \%$ (9/292, Figure 1). Mortality increased from $0 \%$ in RACHS category 1 to $16.7 \%$ (3/18) in RACHS category $5 / 6$ (Table 1 ). Each of the 3 deaths in RACHS categories 5/6 were patients undergoing Norwood procedures who developed severe cardiac dysfunction after cardiotomy with the subsequent need for ECMO, followed by sepsis and renal failure. Of the 150 complications, $37(24.6 \%)$ occurred in the 9 patients who eventually died. Also, 22 of the 150 complications were major adverse cardiac events. Of these 22 major adverse cardiac complications, 10 occurred in patients who died. In the patients who died, the primary complications were cardiac (severe cardiac dysfunction, low cardiac output, unplanned cardiac reoperation, and ECMO), followed by a series of secondary complications (eg, sepsis and renal failure) that eventually culminated in death.

\section{Total Morbidity}

Of the 292 procedures, $84(28.8 \%)$ were associated with a total of 150 complications (average, 0.5 complications/procedure; Table 1). Most procedures with complications (53/84) were associated with a single complication. Multiple complications occurred in 31 procedures (Table 1). Most complications occurred in the RACHS category 3 procedures. Arrhythmias and cardiac and operative complications accounted for $39.7 \%$ of all

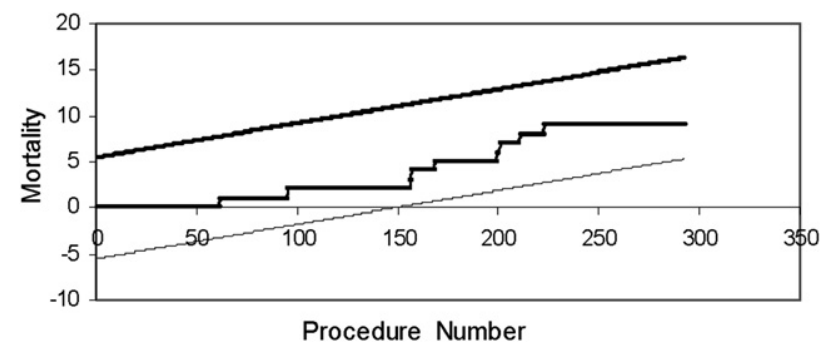

FIGURE 1. Cumulative sum (CUSUM) plot of mortality in the entire cohort. Using $3.5 \%$ as an "acceptable" mortality rate, according to our institutions' historic rates and published data rates of current mortality in pediatric cardiac surgery cohorts, the slope of the curve remains between the alarm limits for the entire duration of the study, implying that program is "in control" with regard to mortality rates. complications (Figure 2). Eight procedures were associated with postcardiotomy ECMO. Of the 150 complications, $16(10.7 \%)$ did not meet the Multi-Societal Committee standardized definitions and were categorized as "other." Included in the "other" category were vascular or conduit thromboses $(\mathrm{n}=3)$, urinary tract infection or intravenous site infection $(n=3)$, gastrointestinal or gastrourinary bleeding $(\mathrm{n}=2)$, hypertension $(\mathrm{n}=2)$, stridor $(\mathrm{n}=2)$, aspiration $(\mathrm{n}=1)$, hepatic dysfunction $(\mathrm{n}=1)$, intussusception $(\mathrm{n}=1)$, and a major skin complication $(\mathrm{n}=1)$.

\section{Complications Stratified by Surgical Complexity and Severity}

A peak was found in the raw frequency of complications and the morbidity burden in the mid-complexity cases (RACHS 3 or ABC level 2 and 3; Figure 3, $A$ and $B$ ).

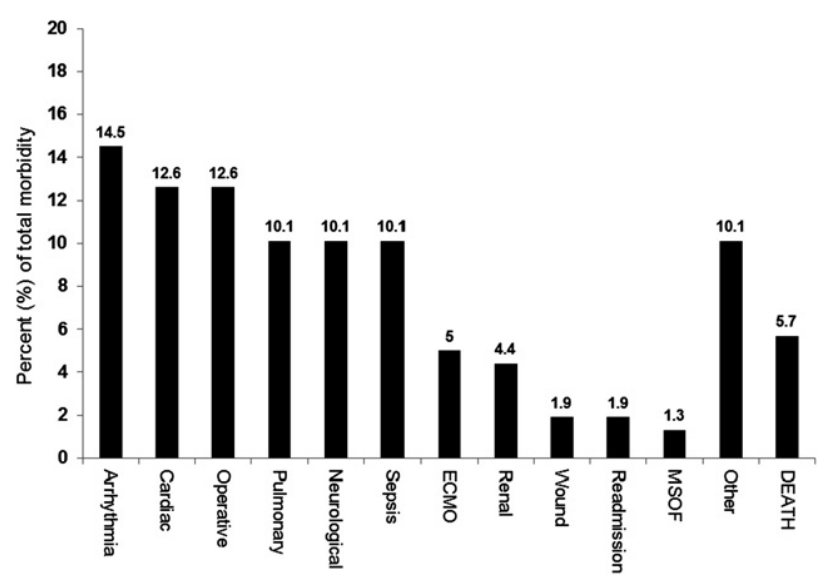

FIGURE 2. Total cohort complications stratified by the organ system. Complications were grouped according to the Multi-Societal Committee for Pediatric and Congenital Heart Disease classification. Most complications in our retrospective analysis were arrhythmias (14.5\%) and cardiac $(12.6 \%)$ and operative $(12.6 \%)$ complications. The 16 complications $(10.1 \%)$ classified as "other" included vascular or conduit thrombosis $(\mathrm{n}=3)$, urinary tract or intravenous site infections $(\mathrm{n}=3)$, gastrointestinal or genitourinary bleeding $(\mathrm{n}=2)$, hypertension associated with recoarctation $(n=2)$, stridor $(n=2)$, aspiration $(n=1)$, hepatic dysfunction $(n=1)$, intussusception $(\mathrm{n}=1)$, and a skin blister secondary to antiseptic skin preparation solution $(\mathrm{n}=1)$. 


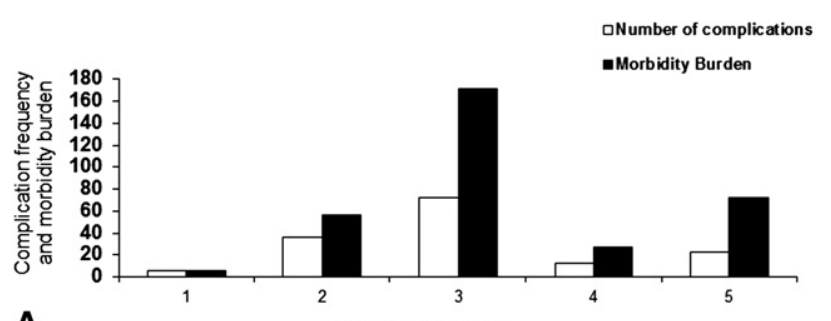

A

RACHS CATEGORY

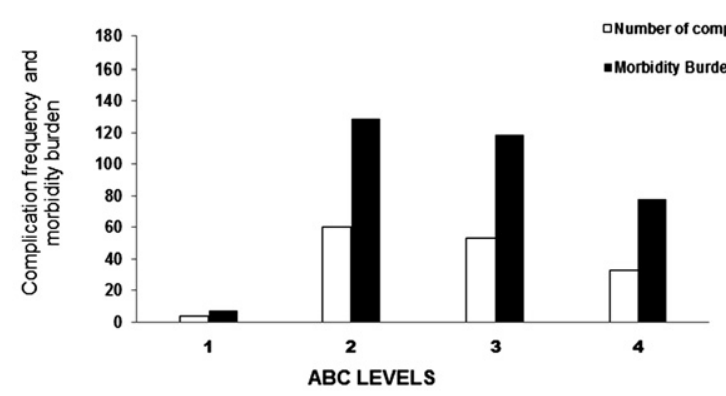

B ${ }^{\star}$ Morbidity burden $=\Sigma$ (frequency $x$ severity )

FIGURE 3. Complications stratified by surgical complexity. The highest complication frequency (white bars) and morbidity burden (black bars; calculated by summing the product of complications multiplied by the severity coefficient for a given surgical stratum) was in (A) Risk Adjustment for Congenital Heart Surgery (RACHS) category 3 and (B) Aristotle basic complexity $(A B C)$ level 2 . These were also the groups with the greatest number of procedures.

A direct linear relationship was found for the morbidity burden indexed to the number of procedures in that stratum of surgical complexity (Figure 4, $A$ and $B$ ). When we analyzed the complications by specific procedure, rather than by RACHS category, a similar linear relationship was found between the indexed morbidity and the complexity of the procedure (data not shown). We then analyzed complications by severity versus surgical complexity. The frequency of severity 3 complications increased from $1.7 \%$ in RACHS category 1 to $61.1 \%$ in RACHS category 6 . The frequency of severity 2 complications demonstrated a similar pattern (RACHS 1, 0\%; RACHS $6,66.7 \%$ ). Severity 1 complications, however, occurred at a rate of $5.2 \%$ and $6.1 \%$ in RACHS 1 and 2 , respectively, but were absent in RACHS categories 4,5 , and 6 (data not shown).

\section{Survivors Versus Nonsurvivors}

To explore the relationship between complications and death, we divided the total cohort into survivors $(n=283)$ and nonsurvivors $(n=9)$. Regardless of the RACHS category, the average indexed morbidity of the nonsurvivors was greater $(10.0 \pm 1.0$; range, $9-11)$ than that of the survivors (1.07 \pm 0.61 ; range, $0.4-1.6)$. The frequency of severity 1 complications in nonsurvivors was threefold that of the survivors. Likewise severity 2 complications occurred 9 times more frequently and severity 3 complications 22
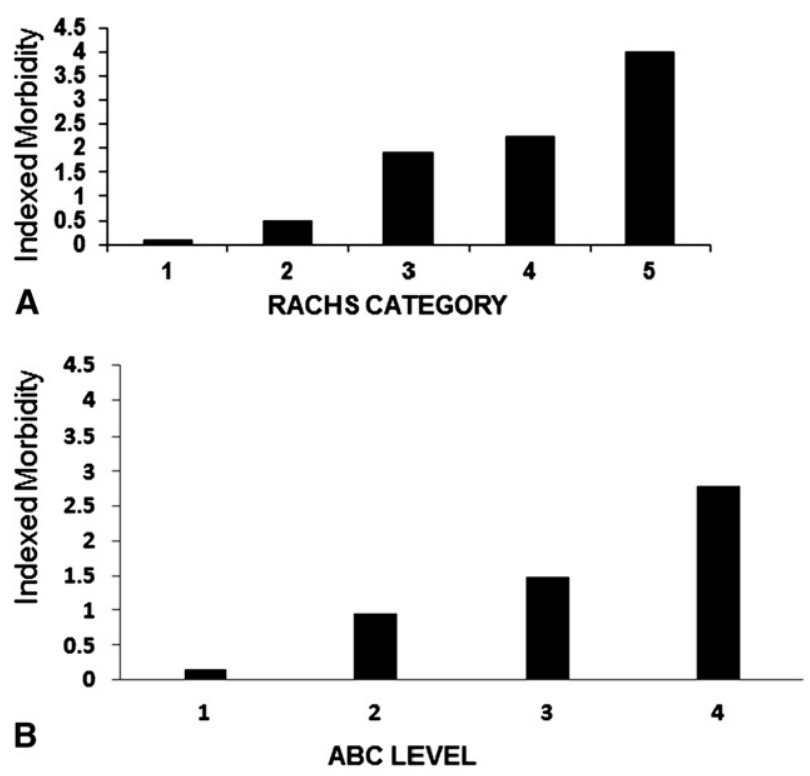

\section{*Indexed morbidity = Morbidity burden / Number of procedures in that RACHS category or ABC level}

FIGURE 4. Risk-stratified complications indexed to the number of procedures in each surgical strata. There was a linear relationship between indexed morbidity (y axis; calculated as $\Sigma$ [complication frequency $\times$ severity coefficient $] /$ number of procedures per surgical strata) and complexity of the procedure (x axis) according to (A) Risk Adjustment for Congenital Heart Surgery (RACHS) category or (B) Aristotle basic complexity $(A B C)$ level.

times more frequently in the nonsurvivors than in the survivors (data not shown).

\section{CUSUM Plots of Morbidity}

We applied the CUSUM method to the analysis of complications in the total cohort (Figure 5, A). The slope of the line was less than that of the control limits such that the lower alarm limit was crossed at patients 90 to 92 . This implies that the complications were accrued at a slower rate than predicted or that the program was outperforming the model after the first third of the series.

To account for the frequency and severity of complications associated with each procedure, we calculated the morbidity burden associated with every procedure in the series and plotted the morbidity burden against the sequential case number (Figure 5, B). The morbidity burden curve (upper curve) demonstrated a much greater slope than the curve that simply plots the number of procedures with any complication (lowest curve). When nonsurvivors were excluded from this analysis, a notable decrease was found in the slope of the curve (middle curve vs upper curve), demonstrating the effect of death on the morbidity burden. The morbidity burden across the entire cohort of 292 procedures was 337 (indexed morbidity, 1.15). The morbidity burden in the 283 survivors was 211 (indexed morbidity, 0.74). 

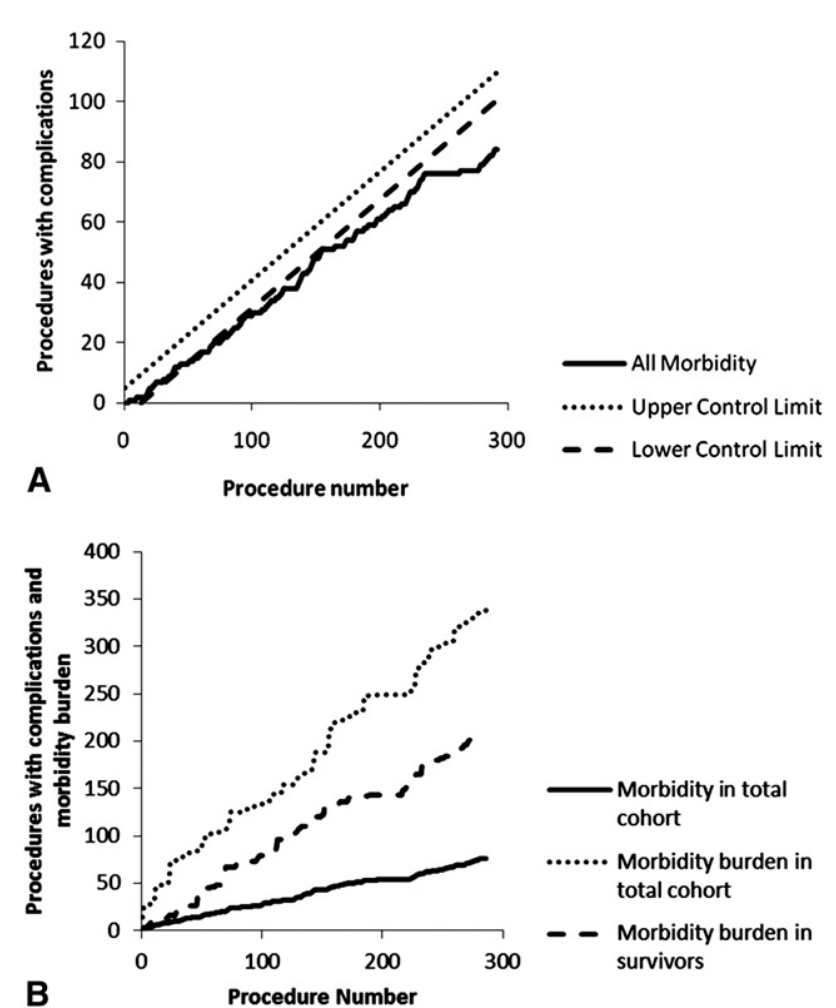

FIGURE 5. Cumulative sum (CUSUM) plot of complications in the total cohort. A, Plotting the occurrence of any complication versus procedure number generates a plot of overall program performance but does not incorporate the concept of frequency and severity into the graphic depiction of outcome. B, Three curves showing the dramatic effect on the slope of the line when one incorporates frequency and severity (morbidity burden in entire cohort) into the reporting of complications (upper curve) compared with simply plotting the occurrence of any complication (morbidity, lowest curve). It also illustrates the effect of including deaths in the analysis (upper curve) compared with excluding deaths from the analysis (morbidity burden in survivors, middle curve).

\section{DISCUSSION}

Historically, operative mortality has been the primary tool for judging performance in congenital cardiac surgery. In the current era, overall congenital cardiac surgical mortality has been reported as $4 \% .{ }^{1}$ We have shown, in the present report, that complications occur at a greater frequency in patients dying but also at reasonably high rates in those not dying as an endpoint. As a result of the distribution of complications, most patient outcomes were unaccounted for when using mortality as the sole indicator of performance in a pediatric cardiac surgical program.

In contrast to mortality, the capture and reporting of complications is relatively difficult. Because of the complexity of defining and accurately reporting complications, there has been some effort to identify surrogate markers of complications. O'Brien and colleagues ${ }^{11}$ chose a prolonged hospital stay ( $>21$ days) as a marker of morbidity, and Clarke and associates ${ }^{12}$ proposed using postoperative length of stay and ventilator duration. Both the length of hospital stay and the ventilation duration have not been validated as surrogates for morbidity. For example, a stay in the hospital of longer than 21 days for a neonate does not necessarily imply the occurrence of a complication because the duration of the preoperative phase is variable, depending on the pathologic features and comorbidities in a given patient. Likewise, postoperative stays are often prolonged for reasons other than operative complications, including feeding, growth, and social issues. The development of a comprehensive list of specific, rather than surrogate, markers has allowed, for the first time, the collection of detailed complication data for patients and cohorts program-wide.

Whether to include mortality in the computation of complications has been debated in published reports. ${ }^{12} \mathrm{We}$ included mortality in our results because we can imagine a scenario in which a surgical program could have low or acceptable complication rates, as a result of having high mortality rates. This apparent contradiction in program performance, depending on whether mortality or morbidity is studied, can be prevented by including deaths in the calculation of the complications. By weighting death with a large severity coefficient, the effect of mortality can be emphasized in a series of complications. We arbitrarily used a severity coefficient of 5 for death, although that might be too small a number to accurately represent the full magnitude of the outcome.

One third of all procedures were associated with complications, a rate consistent with other published reports. ${ }^{7}$ Most of the complications occurred in patients in RACHS category 3 (ABC level 2 ) and greater. However, merely documenting the occurrence or frequency of complication occurrence does not convey the effect of complications on an individual patient or a patient cohort. For example, 5 wound infections in any particular surgical complexity stratum are likely to have a very different net effect on the patient population than 5 ECMO occurrences in the same patient group. Likewise, the effect of stroke or renal failure on overall outcome and quality of life clearly differs from the effect of a superficial wound infection. Thus, along with frequency of occurrence, there must be some way to factor severity into the reporting of complications. We incorporated complication severity into our system by assigning a severity coefficient (range, 1-3) based on the expert consensus of how likely (or not) a particular complication is to predate death (with death assigned a severity coefficient of 5). Although there can be debate about what actual number should be assigned as a coefficient, with large enough data sets, these coefficients will be generated by the data themselves.

To risk stratify complications, which has not been undertaken in published studies before our report, surgical complexity was invoked. The morbidity burden in each surgical complexity stratum was calculated by summing 
the product of each individual complication multiplied by its severity coefficient. The frequency and severity of complications, when indexed to the number of procedures in each group, correlated linearly with increasing surgical complexity. It is intuitive that more complex procedures would be associated with greater rates of complications, but this has not been reported before the present study.

When the total population was divided into survivors and nonsurvivors, the odds of developing a complication of any severity was much greater in the nonsurvivors. When a comparative analysis between survivors and nonsurvivors was performed, only the complications (excluding death) were reported for each patient. A similar indexed morbidity was associated with death, regardless of the surgical complexity, implying that a similar amount of injury had to be accrued before a patient died, regardless of the complexity of the patient's underlying pathologic features.

\section{CUSUM Method to Report Adverse Outcomes}

The CUSUM method is a simple, graphic display of performance that was first used in quality assurance in industrial manufacturing and has since applied to the analysis of adverse occurrences in medicine. ${ }^{13}$ The slope of the line on a CUSUM plot reflects the occurrence of adverse events; the greater the slope of the line, the more frequent the occurrence of adverse events. ${ }^{14}$ In 1994, de Leval and colleagues ${ }^{15}$ presented a CUSUM analysis of mortality in a surgical series of arterial switches. Since then, studies have shown that CUSUM plots are more sensitive than standard statistical tests (eg, chi-square test) in identifying a cluster of failures. ${ }^{16}$ Although CUSUM charts are simple to construct, care is needed to avoid overinterpreting or misinterpreting them. ${ }^{10}$ Also, although CUSUM plots provide a method of displaying data, they are not tests of statistical significance; however, not all instances of deteriorating performance need statistical proof to merit attention. When the CUSUM curve reaches either the upper or lower alarm limit, the process is said to be "out of control"; however, the CUSUM method does not have the discriminatory ability to identify systems that are destabilizing or "going out of control." With a CUSUM analysis, there is a tradeoff in the choice of control limit. A broad limit provides protection against false signals resulting from chance but will be slow to detect a change in process performance, and a narrow control limit will detect changes quickly but will provide frequent false signals. In contrast to mortality, there is a lack of objective comparator data with regard to complications, making it difficult to set control limits on a morbidity CUSUM curve. The CUSUM method has conventionally been used to track mortality. We extended the use of the CUSUM method to the analysis of complications. Because this curve reflects each procedure that is associated with at least 1 complication, it is not currently designed to show the number of complications associated with each procedure or the severity of those complications. CUSUM analysis of the morbidity burden for each procedure (the sum of the severity coefficients for each complication associated with that procedure), in addition to the number of procedures with a complication, addresses this issue and accounts for both the frequency and the severity of every complication associated with each procedure and gives us a better estimate of the effect of the complications associated with each procedure. Furthermore, it is possible to track complications in the total cohort, as well as in survivors, allowing us to estimate the effect of death on the morbidity burden.

The concept of morbidity burden, coupled with the versatility of the CUSUM method, has enabled us to develop a reporting system that not only estimates and tracks the burden of complications in a given patient cohort but also tackles the confounding effect of death on the complications. As with surgical mortality, the relationship of complications to surgical volume remains uncertain and, given the small numbers of cases used to generate the present retrospective data, using the absolute morbidity data we have reported is not necessarily representative for comparison with other programs.

The CUSUM plots should ideally be risk adjusted to account for variations in preoperative risk to "level the playing field." We used the CUSUM method in the context of pediatric cardiac surgery to track total complications and complications stratified by severity. CUSUM plots could also be used to track complications by specific procedure (eg, Norwood procedure), specific complication (eg, arrhythmia), or specific RACHS category. This is a method in evolution and will undergo additional refinement as more objective data become available.

\section{Study Limitations}

The retrospective design of the study resulted in inherent limitations. Retrospective data capture could be associated with an underreporting of complication frequency. The small numbers of procedures in some groups hampered subset analysis. Specifically, our small volume prevented any meaningful calculation of disease-specific or operationspecific complication rates. Furthermore, data from the present retrospective single small-volume center should not be overinterpreted and might not be representative for comparison with other programs. As with surgical mortality, the relationship of complications to surgical volume remains uncertain.

The absence of existing published data or benchmarks for program-wide complications means that we can only compare our results with our own historic data until such time as other institutional data becomes available. This circular reference could be misleading, in that staying within alarm limits only reflects current outcomes; thus, if these outcomes are poor to start with, staying within the alarm limits would be falsely reassuring. Once multicenter prospective 
data become available, the alarm limits will be recalculated, and our data can be benchmarked against more objective comparators. Our reporting only included pediatric cardiac surgical procedures and not adult patients with congenital heart disease.

\section{CONCLUSIONS}

When examined in a systematic fashion, the risk of complications in pediatric cardiac surgical patients is considerable. Our data illustrate that it is possible to track complications over time in a consistent manner. The effect of complication monitoring on patient outcomes remains to be proved.

The authors gratefully acknowledge Daniel Belliveau and Trudy Hennigar for their assistance and Karen Buth for her statistical consultation.

\section{References}

1. Jacobs JP, Maruszewski B, Tchervenkov CI, Lacour-Gayet FG, Jacobs ML, Clarke DR, et al. The current status and future directions of efforts to create a global database for the outcomes of therapy for congenital heart disease. Cardiol Young. 2005;15(Suppl 1):190-7.

2. Jacobs JP, Jacobs ML, Mavroudis C, Maruszewski B, Tchervenkov CI, LacourGayet FG, et al. What is operative morbidity? Defining complications in a surgical registry database. Ann Thorac Surg. 2007;84:1416-21.

3. Ferraris VA, Ferraris SP. Risk factors for postoperative morbidity. J Thorac Cardiovasc Surg. 1996;111:731.

4. Hammermeister KE, Burchfiel C, Johnson R, Grover FL. Identification of patients at greatest risk for developing major complications at cardiac surgery. Circulation. 1990;82(5 Suppl):IV380-9.
5. Silber JH, Rosenbaum PR, Schwartz JS, Ross RN, Williams SV. Evaluation of the complication rate as a measure of quality of care in coronary artery bypass graft surgery. JAMA. 1995;274:317-23.

6. Shroyer AL, Coombs LP, Peterson ED, Eiken MC, DeLong ER, Chen A, et al. The Society of Thoracic Surgeons: 30-day operative mortality and morbidity risk models. Ann Thorac Surg. 2003;75:1856-64.

7. Benavidez OJ, Gauvreau K, Del Nido P, Bacha E, Jenkins KJ. Complications and risk factors for mortality during congenital heart surgery admissions. Ann Thorac Surg. 2007;84:147-55

8. Jenkins KJ, Gauvreau K, Newburger JW, Spray TL, Moller JH, Iezzoni LI. Consensus-based method for risk adjustment for surgery for congenital heart disease. J Thorac Cardiovasc Surg. 2002;123:110-8.

9. Lacour-Gayet F, Clarke D, Jacobs J, Comas J, Daebritz S, Daenen W, et al. The Aristotle score: a complexity-adjusted method to evaluate surgical results. Eur J Cardiothorac Surg. 2004;25:911-24.

10. Rogers CA, Reeves BC, Caputo M, Ganesh JS, Bonser RS, Angelini GD. Control chart methods for monitoring cardiac surgical performance and their interpretation. J Thorac Cardiovasc Surg. 2004;128:811-9.

11. O'Brien SM, Jacobs JP, Clarke DR, Maruszewski B, Jacobs ML, Walters HL III, et al. Accuracy of the Aristotle basic complexity score for classifying the mortality and morbidity potential of congenital heart surgery operations. Ann Thorac Surg. 2007;84:2027-37.

12. Clarke DR, Lacour-Gayet F, Jacobs JP, Jacobs ML, Maruszewski B, Pizarro C, et al. The assessment of complexity in congenital cardiac surgery based on objective data. Cardiol Young. 2008;18(Suppl 2):169-76.

13. Novick RJ, Fox SA, Stitt LW, Forbes TL, Steiner S. Direct comparison of risk-adjusted and non-risk-adjusted CUSUM analyses of coronary artery bypass surgery outcomes. J Thorac Cardiovasc Surg. 2006;132: 386-91.

14. Williams SM, Parry BR, Schlup MM. Quality control: an application of the CUSUM. BMJ. 1992;304:1359-61.

15. de Leval MR, François K, Bull C, Brawn W, Spiegelhalter D. Analysis of a cluster of surgical failures: application to a series of neonatal arterial switch operations. J Thorac Cardiovasc Surg. 1994;107:914-23.

16. Novick RJ, Fox SA, Stitt LW, Kiaii BB, Swinamer SA, Rayman R, et al. Assessing the learning curve in off-pump coronary artery surgery via CUSUM failure analysis. Ann Thorac Surg. 2002;73:S358-62. 